\title{
Os desafios na sistematização da assistência de enfermagem em serviços de urgência e emergência
}

\author{
The challenges in systematizing reference assistance in emergency and emergency \\ services

\section{Los desafíos en la asistencia de referencia sistematizante en servicios de emergencia y de emergencia}

\begin{abstract}
Maria Lara Costa Manso ${ }^{1 *}$, Rebeca Larissa Nepomuceno Torres ${ }^{1}$, Mateus Ferreira dos Santos ${ }^{1}$, Maria Eliane Barbosa de Araújo ${ }^{1}$, Amanda Luana Lopes da Silva1, Arthur Victor Carvário de Farias $^{1}$, Daniela Barbosa de Lima ${ }^{1}$, Cíntia de Carvalho Silva ${ }^{1}$, Lidiane Marinho da Silva Barbosa ${ }^{1}$, Diego Augusto Lopes Oliveira'.
\end{abstract}

\section{RESUMO}

Objetivo: Descrever os obstáculos existentes nos serviços de urgências e emergências que impedem a implementação da sistematização da assistência de enfermagem. Métodos: Trata-se de um estudo descritivo do tipo revisão integrativa, o qual foi norteado pela questão sobre quais são os obstáculos existentes nos serviços de urgências e emergências que impedem a implementação da sistematização da assistência de enfermagem. Utilizou-se o método de busca avançada por título, resumo e assunto, considerando-se as publicações dos últimos dez anos. Resultados: Com emprego dos descritores e a aplicação dos critérios de elegibilidade foram encontradas 182 publicações, após a leitura de títulos, resumo e íntegra, foram selecionados 03 artigos. Evidenciou-se diversas causas que interferem na consolidação das ações de sistematização da assistência de enfermagem, sendo: à resistência e comodismo dos profissionais, ausência de instrumentos específicos para esse setor, falta de tempo da equipe, escassez de conhecimento sobre a operacionalização do método de sistematização e o inadequado dimensionamento dos profissionais, implicando no déficit de registros nos prontuários dos pacientes e difículta a continuidade da assistência. Considerações finais: A implementação da sistematização da assistência de enfermagem contribui o cuidado ofertado nos serviços de urgência e emergência, contudo ainda há dificuldades para essa implantação nesses serviços.

Palavras-chave: Cuidados de enfermagem, Emergência, Processo de enfermagem, Serviço hospitalar de emergência, Socorro de urgência.

\section{ABSTRACT}

Objective: To describe the obstacles in urgent and emergency services that prevent the implementation of the systematization of nursing care. Methods: This is a descriptive study of the integrative review type, which was guided by the question of what obstacles exist in urgent and emergency services that prevent the implementation of the systematization of nursing care? The advanced search method by title, abstract and subject was used, considering the publications of the last ten years. Results: With the use of descriptors and the application of eligibility criteria, 182 publications were found, after reading the titles, abstract and full text, 03 articles were selected. Several causes were evidenced that interfere in the consolidation of the actions of systematization of nursing care, such as: the resistance and convenience of professionals, absence of specific instruments for this sector, lack of team time, lack of knowledge about the operationalization of the method of systematization and inadequate sizing of professionals, resulting in a deficit of records in patients' medical records and hindering the continuity of care. Final considerations: The implementation of the systematization of nursing care contributes to the care offered in urgent and emergency services, however there are still difficulties for this implementation in these services.

Keywords: Nursing care, Emergency, Nursing process, Emergency hospital service, Urgency relief.

\section{RESUMEN}

Objetivo: Describir los obstáculos en los servicios de urgencia y emergencia que impiden la implementación de la sistematización de la atención de enfermería. Métodos: Se trata de un estudio descriptivo del tipo revisión integradora, el cual fue guiado por la pregunta ¿qué obstáculos existen en los servicios de urgencia y emergencia que impiden la implementación de la sistematización de la atención de enfermería? Se utilizó el método de búsqueda avanzada por título, resumen y tema, considerando las publicaciones de los últimos diez años. Resultados: Con el uso de descriptores y la aplicación de criterios de elegibilidad, se encontraron 182 publicaciones, luego de la lectura de títulos, resumen y texto completo, se seleccionaron 03 artículos. Se evidenciaron varias causas que interfieren en la consolidación de las acciones de sistematización del cuidado de enfermería, tales como: la resistencia y conveniencia de los profesionales, ausencia de instrumentos específicos para este sector, falta de tiempo en equipo, desconocimiento sobre la operacionalización del método. de sistematización y dimensionamiento inadecuado de los profesionales, lo que genera un déficit de registros en la historia clínica de los pacientes y dificulta la continuidad de la atención. Consideraciones finales: La implementación de la sistematización de la atención de enfermería contribuye a la atención ofrecida en los servicios de urgencias y emergencias, sin embargo, aún existen dificultades para esta implementación en estos servicios.

Palabras clave: Atención de enfermería, Emergencia, Proceso de enfermería, Servicio de emergencia hospitalaria, Alivio de urgencia.

\footnotetext{
${ }^{1}$ Centro Universitário Tabosa de Almeida (ASCES-UNITA), Caruaru - PE. *E-mail: laramlcm@hotmail.com
} 


\section{INTRODUÇÃO}

O cenário brasileiro dos serviços de urgência e emergência no país evidenciam elevadas taxas de morbimortalidade devido a causas externas, decorrentes principalmente de violência e acidentes de trânsito, sendo essa realidade responsável por cerca de $50 \%$ das mortes em todo o mundo a cada ano. Estudos apontam que estes setores apresentam altas taxas de ocupação e baixa rotatividade de pacientes, quando associados ao dimensionamento inadequado acarreta em sobrecarga dos trabalhadores. Nessa perspectiva, verifica-se a necessidade de organização desses setores, para o atendimento a esse público que se encontra vulnerável e gravemente ferido, a fim de proporcionar uma assistência qualificada, aplicando ações de prevenção, promoção e recuperação da saúde, prezando pela segurança dos usuários (ANDRADE PM, et al., 2019; GOMES ATL, et al., 2019).

Por se tratar de unidades nas quais as demandas ocorrem sem programação prévia e são envolvidas por situações de estresse, em meio às condições adversas que permeiam o cenário dos serviços de urgência e emergência do Brasil, grande parte dos profissionais enfrentam circunstâncias desafiadoras como, o dimensionamento inadequado de recursos humanos em enfermagem, falta estabelecimento de prioridades nos atendimentos e demanda excessiva de trabalho. Nesta perspectiva é notória a necessidade de padronizar um Processo de Enfermagem (PE) prático com o objetivo de colher o máximo de informações possíveis, substituindo a triagem excludente por um modelo acolhedor que irá contribuir com 0 desenvolvimento das práticas de enfermagem e para o planejamento de um cuidado adequado para 0 paciente (MIRANDA CA, et al., 2012; SERRA HHN, et al., 2019; SOKOLSKI LB, et al., 2019).

Neste contexto, o processo de trabalho, a modalidade de atenção e o perfil de morbidade populacional levam a reflexão sobre a forma do desenvolvimento do cuidado nos serviços de média e alta complexidade, desta forma, a enfermagem tem papel fundamental na elaboração do PE nos serviços de urgência e emergência, a qual é uma atividade exclusiva do profissional enfermeiro que prestará cuidados durante a hospitalização do paciente, realizando uma avaliação de acordo com a sua singularidade e de forma integral, levando em consideração não só as necessidades biológicas, como as espirituais e sociais, permitindo que ocorra um cuidado integral e contínuo (NICOLAU S, et al., 2019).

A Sistematização da Assistência de Enfermagem (SAE) é um importante instrumento metodológico de gerenciamento do cuidado, que ao ser executado de forma eficiente promove diversos benefícios para os setores, dentre os quais se podem destacar: um fluxo de atendimento mais organizado, maior facilidade nas passagens de plantões, assistência segura e baseada em evidências cientificas, controle de gastos, otimização do tempo, maior autonomia e um dos principais meios de valorização dos profissionais de enfermagem, além de um meio de fortificar a interação entre o profissional, usuário e família (MALVESTIO MAA, et al., 2019).

O PE é a representação maior do método científico da profissão, havendo como objetivo a prestação de cuidados de enfermagem baseados em evidências científicas, sendo organizado pela SAE, a qual é instituída pela resolução no 358/2009 do Conselho Federal de Enfermagem (COFEN) e regulamentada pela Lei do exercício Profissional $n^{\circ} 7489$ de 25 de junho de 1986, estabelecendo que seja implementada em todas as unidades de atendimento de saúde, inclusive nos serviços de urgência e emergência de qualquer instituição seja pública ou privada que realizem os cuidados de enfermagem. Esta sistematização permite que sejam avaliadas as condições e necessidades de cada paciente, orientando o profissional à prática de uma assistência ordenada e registrada de maneira lógica (MARIA MA, et al., 2012; SOARES MI, et al 2015).

O arcabouço teórico do PE compreende cinco etapas: anamnese e exame físico, diagnóstico de enfermagem, planejamento e implementação da assistência e avaliação. Esse processo é realizado de forma autônoma pelo enfermeiro, permitindo raciocínio e acurácia das ações e cuidados a serem prestados, o que proporciona poder de decisão para atender de forma sistemática e individualizada as demandas originadas pelos usuários, ademais essa prática norteia desenvolvimento do pensamento crítico, contribui para tomada de decisões da equipe de enfermagem e favorece novas maneiras de gerenciamento mesmo nos setores de urgência e emergência que demandam agilidade e maior capacidade resolutiva, uma vez que a dinâmica de gerenciar, cuidar e educar pode permear e conectar os serviços hospitalares (SANTOS WN, 2014; OLIVEIRA RJT, et al., 2015). 
Neste contexto, considerando a realidade dos atendimentos de média e alta complexidade no Brasil, os benefícios oriundos de uma assistência de enfermagem sistematizada para este tipo de serviço, e com intuito de promover um atendimento eficiente e de qualidade ao paciente, o presente estudo tem como objetivo descrever os desafios e dificuldades enfrentados pela equipe de enfermagem para implementação da sistematização da assistência de enfermagem nos serviços de urgências e emergências.

\section{MÉTODOS}

Trata-se de um estudo bibliográfico, descritivo, do tipo revisão integrativa de literatura, no qual a busca nas bases de dados norteou-se por meio da questão sobre quais são os obstáculos existentes nos serviços de urgências e emergências que impedem a implementação da sistematização da assistência de enfermagem. A qual foi desenvolvida através da estratégia PICO, que representa um acrônimo para Paciente, Intervenção, Comparação e Outcomes (desfecho), se caracterizando como elementos essenciais para a construção da pergunta norteadora para a busca bibliográfica (LATORRACA COC, et al., 2019)

Adotaram-se as seguintes etapas metodológicas para construção do estudo: Inicialmente se realizou a identificação do tema e elaboração da pergunta que norteou a pesquisa, após foram estabelecidos os critérios para inclusão e exclusão de estudos na literatura, em seguida se fez a estruturação das informações a serem coletados nos estudos elegidos, posteriormente os estudos eleitos foram examinados e seus resultados interpretados, para finalizar, realizou-se a síntese e apresentação das informações (SOUSA LMM, et al., 2017).

Elencaram-se como ambiente de pesquisa, a base de dados Latino-Americana em Ciências de Saúde (LILACS), utilizando-se o método de busca avançada categorizado por título, resumo e texto completo. Coletaram-se os dados durante o período de 16 a 17 de fevereiro de 2021, considerando-se as publicações dos anos de 2016 a 2020, visto que esses dados eram os mais recentes nas bases de dados. Utilizaram-se os seguintes Descritores em Ciências da Saúde (DeCS): processo de enfermagem e enfermagem em emergência, a partir da associação do operador booleano "AND" na base de dados referida.

Consideraram-se os seguintes critérios de elegibilidade para a análise: artigos publicados em português, inglês e espanhol contendo texto completo, sendo excluídos da pesquisa todos os achados que não estivessem dentro da temática proposta, literaturas duplicadas, artigos de revisão de literatura, teses, dissertações, trabalhos de conclusão de curso, editoriais, resumos e carta de opinião. Ademais, desconsideram-se, para cada etapa, os estudos que não apresentaram coerência com o objetivo da revisão, bem como os que não estivessem de acordo com os critérios estabelecidos para a seleção amostral.

Os estudos foram classificados de acordo com a hierarquia de evidências apresentadas, no qual pode-se considerar sete níveis, sendo o primeiro: revisões sistemáticas ou metanálises, o segundo: estudos controlados e aleatórios, o terceiro: estudos controlados sem randomização, o quarto: estudos caso-controle ou de coorte, o quinto: revisões sistemáticas de estudos qualitativos ou descritivos, o sexto: estudos qualitativos ou descritivos e no ultimo nivel: estudos de opiniões ou consensos (FERREIRA VHS, et al., 2019).

A avaliação dos manuscritos foi realizada no mês de maio de 2021 de forma crítica e minuciosa adotando o processo de revisão por pares, com o intuito de minimizar erros na seleção, seguindo o roteiro para a análise dos estudos: I-Leitura do título da publicação selecionando aqueles que apresentavam relação com o objetivo desta pesquisa; II- Leitura crítica e exploratória dos resumos, observando-se os critérios de inclusão; III- leitura do texto completo na integra. A seleção da publicação foi elaborada de acordo com Preferred Reporting Items for Systematic Reviews and Meta-Analyses (PRISMA) (MOHER D et al., 2015)

Reuniu-se, após a leitura crítica dos artigos, um produto de análise crítica e interpretação da leitura para o estudo. Realizou-se, como última etapa, a apresentação das pesquisas, por meio da discussão dos resultados relevantes na literatura, possibilitando a realização da análise crítica dos estudos e a inclusão de temáticas importantes referentes à pesquisa. 


\section{RESULTADOS}

A partir do cruzamento dos descritores se obteve 495 artigos, após a aplicação dos critérios de elegibilidade permaneceram 182 publicações. Excluíram-se 176 artigos após a aplicação dos critérios de exclusão e leitura de títulos e resumos, resultando em 06 publicações. Excluíram-se, ainda, após a leitura na íntegra, 3 por não compactuar com o objetivo do estudo ou não responderem à questão norteadora, perdurando 3 estudos para a construção desta revisão integrativa, onde cada artigo atendeu aos critérios de inclusão previamente estabelecidos. Salienta-se que, dentre os três artigos incluídos na revisão integrativa, todos foram de autoria de enfermeiros.

Publicaram-se os estudos selecionados no período de 2016 a 2019, em revistas nacionais. Informa-se que o descritor mais utilizado pelos autores dos artigos foi Processo de Enfermagem, o qual destaca-se por ser de grande relevância em linhas de pesquisa de diversos autores. Constatou-se durante o delineamento das informações que dois artigos apresentavam-se com abordagem metodológica qualitativa e um com abordagem quantitativa. Ilustra-se, na Figura 1 o processo de seleção dos artigos avaliados, já os principais achados dos artigos incluídos para este estudo estão dispostos no Quadro 1.

Figura 1 - Processo de seleção dos artigos durante a de coleta dos dados.

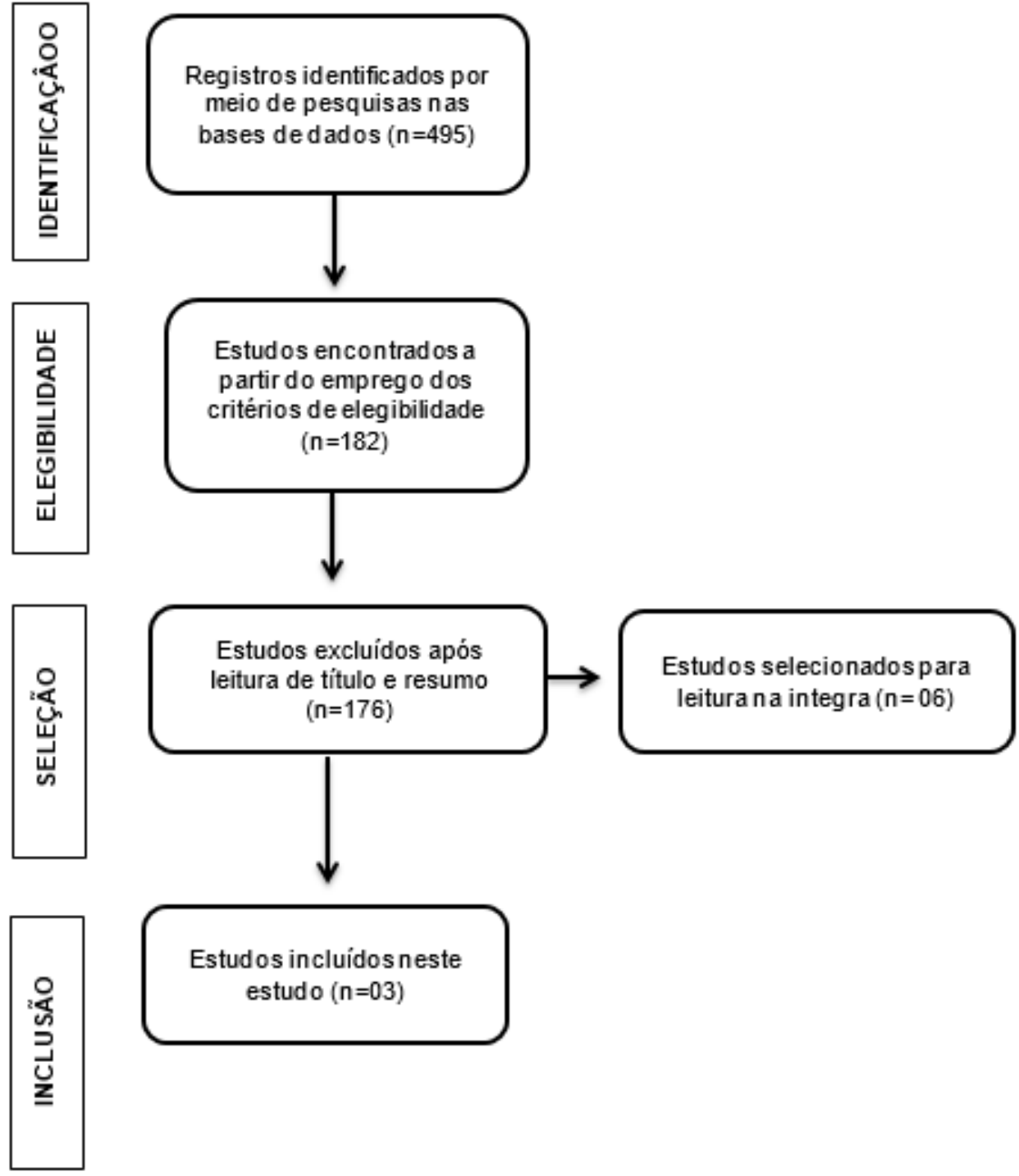

Fonte: Manso MLC, et al., 2021.

Evidencia-se no Quadro 1, os artigos avaliados representados por autores, título e ano da publicação, nível de evidência e principais achados. 
Quadro 1 - Caracterização dos artigos selecionados para a descrição dos desafios e das dificuldades que a equipe enfrenta para implementar a sistematização da assistência de enfermagem nos serviços de urgência e emergência.

\begin{tabular}{|c|c|c|c|c|}
\hline ID & $\begin{array}{c}\text { Autores } \\
\text { (Ano) }\end{array}$ & $\begin{array}{c}\text { Nível de } \\
\text { evidência }\end{array}$ & Título & Principais achados \\
\hline 1 & $\begin{array}{l}\text { MARCOS } \\
\text { ACA, et al. } \\
(2016)\end{array}$ & IV & $\begin{array}{l}\text { Percepção da equipe de enfermagem } \\
\text { quanto a sistematização da assistência de } \\
\text { enfermagem em um serviço de emergência } \\
\text { psiquiátrica. }\end{array}$ & $\begin{array}{l}\text { Trata-se de estudo qualitativo transversal de caráter descritivo e } \\
\text { exploratório. A SAE no setor de emergência apresenta desafios para sua } \\
\text { adesão por parte de alguns profissionais, sendo os principais: o } \\
\text { comodismo ou resistência em aderir às mudanças, dificuldades dos } \\
\text { funcionários em adotar a novas tecnologias, passividade da chefia, } \\
\text { inadequação de instrumentos e falta de capacitação da equipe sobre a } \\
\text { temática. }\end{array}$ \\
\hline 2 & $\begin{array}{l}\text { CORDEIRO } \\
\text { TLR, et al. } \\
(2019)\end{array}$ & IV & $\begin{array}{l}\text { Prontuário eletrônico como ferramenta para } \\
\text { a sistematização da assistência de } \\
\text { enfermagem no } \\
\text { urgência/emergência: } \\
\text { enfermeiros. }\end{array}$ & $\begin{array}{l}\text { Trata-se de uma pesquisa descritiva, exploratória, com abordagem } \\
\text { qualitativa. O registro do processo de enfermagem no prontuário do } \\
\text { paciente é o que permite a continuidade da assistência, no entanto, } \\
\text { enfermeiros apontam desafios para sua implantação, como: à sobrecarga } \\
\text { de atividades, alta rotatividade de pacientes, acúmulo de tarefas, falta de } \\
\text { informações sobre o paciente, mudança frequentes em suas } \\
\text { necessidades e a escassez de recursos humanos e materiais. }\end{array}$ \\
\hline 3 & $\begin{array}{l}\text { NICOLAU S, } \\
\text { et al. (2019) }\end{array}$ & IV & $\begin{array}{lrrr}\text { Implementação da assistência } & \text { de } \\
\text { enfermagem (SAE) no serviço } & \text { de } \\
\text { atendimento móvel de urgência (SAMU) }\end{array}$ & $\begin{array}{l}\text { Trata-se de um estudo descritivo, com uma abordagem quantitativa, do } \\
\text { tipo transversal. Apesar dos enfermeiros entrevistados, considerarem } \\
\text { que a sistematização da assistência de enfermagem é importante para } \\
\text { uma assistência qualificada, eles não conseguem realizá-la ou não } \\
\text { realizam como pedem as normas da Resolução } 358 \text { do Conselho Federal } \\
\text { de Enfermagem, seja por falta de tempo, pelo desconhecimento da } \\
\text { sistematização, ou pela descontinuidade do processo. }\end{array}$ \\
\hline
\end{tabular}

Fonte: Manso MLC, et al., 2021. 


\section{DISCUSSÃO}

A partir das análises dos artigos foi possível verificar a existência de diferentes fatores que implicam em desafios para a implementação da SAE nos serviços de urgência e emergência. Uma das causas elencadas são a resistência e o comodismo da equipe de enfermagem em adotar mudanças estabelecidas pelos gestores, no qual evidencia-se que os profissionais que realizam a assistência, na maioria das vezes, apresentam relutância em relação às ações propostas por acharem que não contribui com a melhoria da qualidade do setor. Desta forma, se faz necessário que os líderes dos setores sensibilizem e motivem os demais profissionais sobre a importância do PE, para que haja a implementação das ações de forma crítica e consciente, com vistas a proporcionar um cuidado individualizado e sistematizado aos usuários (MARSICO EFC, et al., 2012; REIS GAX, et al., 2019).

Dentre os achados observados, destaca-se que para alcançar êxito na inserção da SAE é essencial que o enfermeiro se posicione frente à equipe, demonstrando que esse método além de proporcionar uma ampliação da situação de saúde do paciente, promove uma organização no fluxo do serviço que irá beneficiar toda a equipe. Nesta perspectiva, o líder deve propiciar o aumento da interação e cooperação, prestando apoio e estimulando os profissionais que atuam nos serviços de urgência e emergência a aderirem às mudanças estabelecidas, uma vez que, o mesmo detém da responsabilidade de encorajar e impulsionar mudanças no ambiente de trabalho, bem como de despertar a autonomia, co-responsabilização e a valorização, com intuito de atingirem os objetivos em comum e melhorar a qualidade da assistência (ANDRADE PM, et al., 2019).

Percebe-se que os profissionais de enfermagem nem sempre detém o conhecimento suficiente acerca de todas as etapas a serem realizadas, refletindo lacunas no processo de formação ainda na graduação, tornando-se um fator prejudicial para os processos organizacionais e tomada de decisão. Nota-se que a enfermagem moderna utiliza o conhecimento científico para o planejamento da assistência, rompendo com o modelo assistencial biomédico e trazendo modelos alternativos, o qual proporciona o cuidado direcionado às necessidades do paciente de forma rápida e autônoma, em virtude das características do setor. Desta forma, além do contínuo treinamento e estudo para aperfeiçoamento das técnicas, é imprescindível o conhecimento e domínio da SAE (NICOLAU S, et al., 2019; MARIA MA, et al., 2012).

Dentre os fatores que interferem na realização da SAE, a sobrecarga de atividades é a mais referida, apontada como um dos motivos que dificultam o processo de trabalho, uma vez que as unidades de urgência e emergência apresentam grande agitação, superlotação e a livre demanda no acolhimento dos pacientes, o que resulta em sobrecarga e falta de tempo dos profissionais para implementar as etapas da sistematização do cuidado. Desta forma, quando o enfermeiro não consegue realizar todas as etapas do PE sua assistência é aplicada de maneira fragmentada, essa situação é decorrente na maioria das vezes pela falta de tempo existente para avaliar todos os pacientes utilizando a SAE, este fato acarreta muitas vezes a necessidade de priorizar os pacientes mais graves, centrando o cuidado na gravidade da patologia (NICOLAU S et al., 2019; PINTO DM, et al., 2021).

Outro fato evidenciado é que por vezes as prescrições dos cuidados acabam sendo executadas apenas verbalmente, sem a realização dos seus registros, o que pode levar à fragilização do cuidado, visto que, os registros de enfermagem contribuem para planejamento e avaliação da assistência, servem como instrumentos para a avaliar o que foi utilizado durante as auditorias e são meios de defesa dos profissionais de saúde em situações de incriminação, uma vez que a emergência o risco iminente à vida é presente e o valor legal de um cuidado prestado é baseado nas anotações, necessitando assim que os registros supervisionados e incentivadas visando à proteção dos profissionais e pacientes (CORDEIRO TLR, et al., 2019; NICOLAU S, et al., 2019; PINTO DM, et al., 2021)

O dimensionamento inadequado dos profissionais é outro fator que contribui com as dificuldades para implementar ou realizar a SAE em todas as suas etapas nos serviços de urgência e emergência, visto que, o perfil do atendimento nesses serviços demanda maior tempo e atenção da equipe de enfermagem, uma vez que a maioria dos pacientes que se encontram nesses setores são idosos e com comorbidades instaladas. Essa situação ocasiona excesso de atribuições para a equipe e expõe os profissionais a estresses físicos e psicológicos e ao risco de doenças ocupacionais, o que possibilita a ocorrência de erros e falhas durante a realização da assistência, contribuindo com o agravo à saúde do usuário e 
consequentemente reflete de forma prejudicial na qualidade do atendimento (REIS GAX, et al., 2019; PAIXÃO TCR, et al., 2015).

Ao abordar a SAE, por meio do Prontuário Eletrônico do Paciente (PEP), subentende-se que os registros de enfermagem ocorram em todas as suas fases, devendo ser registradas todas as etapas inerentes ao PE. No entanto, além das vantagens do PEP para a SAE, também se identifica dificuldades para que todas as fases necessárias sejam colocadas em prática, devido às características distintas dos serviços de urgência e emergência, os pacientes permanecerem por um limitado intervalo de tempo e associada às situações aonde muitos dos usuários não chegam acompanhados nas unidades, o que contribui para um déficit na coleta e no registro das informações. Essas particularidades configuram-se como obstáculos que colaboram para a desvalorização da categoria profissional e vulnerabilidade de erros (CORDEIRO TLR, et al., 2019; SOARES MI, et al., 2015)

Em consonância à realidade em estudo, no que diz respeito aos recursos humanos, materiais e tecnológicos, grande partes dos hospitais públicos brasileiros sofrem com a escassez destes, resultando na necessidade de realizar adaptações e improvisações que muitas vezes não trazem segurança para os profissionais e pacientes durante os procedimentos assistenciais executados, sendo uma característica que também permeia os serviços de urgência e emergência, e que contribui com o desgaste físico e emocional dos profissionais (SILVA LG, et al., 2012).

Nesse contexto, as dificuldades de acesso aos recursos materiais e tecnológicos essenciais, são apontados como uma problemática que implica no registro da SAE no PEP, uma vez que todos os procedimentos deveriam ser registrados de forma informatizada nos hospitais, no entanto a disponibilidade dos computadores nem sempre conseguem suprir as necessidades da equipe, sujeitando assim muitas vezes a abdicação do registro do PE e estabelecimentos de outras prioridades, uma vez que a falta de recursos humanos também se faz presente nessas instituições (CORDEIRO TLR, et al., 2019).

Outra condição que dificulta a execução da SAE é a inadequação dos instrumentos de trabalho, uma vez que, grande parte não são elaborados de acordo com o perfil epidemiológico da unidade que procede aos atendimentos, impossibilitando a atuação da equipe de forma ágil e eficaz. Esse fato encontra-se de acordo com outros estudos analisados, no qual evidenciam a deficiência dos registros relacionados ao processo de enfermagem e demonstram a falta de protocolos adaptados que englobam as suas etapas, tornando-se um entrave no cotidiano do profissional, visto que, esses instrumentos subsidiam o enfermeiro no reconhecimento das necessidades de organização do cuidado, auxiliam na tomada de decisão e contribuem para o planejamento e sistematização da assistência, na medida em que proporcionam a visão do todo (MARCOS ACA, et al., 2016; SOARES MI, et al., 2015).

Desta forma, pode-se inferir que os enfermeiros sentem a necessidade de atividades de educação e capacitação para que possam aplicar na prática cotidiana a SAE de forma adequada e completa, sobretudo, em serviços de emergência que demandam agilidade e experiência dos enfermeiros. Diante disso, 0 reconhecimento profissional acerca da relevância da SAE, só será possível por meio da divulgação dos benefícios tanto para a equipe de enfermagem, como para os pacientes e a instituição de saúde. Destarte, fica evidente a necessidade de sensibilizar todos os envolvidos acerca da indispensabilidade das ações de enfermagem para a melhoria da assistência, sendo essa ação um passo importante a ser adotado pelos serviços para potencializar a SAE, e desenvolver o cuidado com qualidade, originando resultados positivos na assistência prestada (PINTO DM, et al., 2021).

\section{CONSIDERAÇÕES FINAIS}

O presente estudo permitiu uma ampla descrição acerca dos desafios e das dificuldades que os profissionais de enfermagem enfrentam para implementar e realizar ações da SAE nos serviços de urgência e emergência, no qual destaca-se a resistência e comodismo dos profissionais em aderir as etapas do PE, ausência de instrumentos específicos para esse setor, falta de tempo da equipe devido a alta rotatividade e demanda dos pacientes, escassez de conhecimento sobre a operacionalização da SAE e o dimensionamento inadequado dos profissionais. Neste sentindo, esta revisão contribui para identificar esses desafios a fim de minimiza-los. Ademais, nota-se a escassez de estudos na literatura que tratem da SAE nos serviços de urgência e emergência, fazendo-se necessário o incentivo às ações que estimulem a adoção de práticas baseadas em evidências, com intuito de melhorar a assistência prestada. 


\section{REFERÊNCIAS}

1. ALMEIDA AC, et al. Nursing assistance to the emergency/urgency patient. Journal of Nursing UFPE/Revista de Enfermagem UFPE, 2018; 12: 12.

2. ANDRADE PM, et al. Sistematização da assistência de enfermagem: vantagens e dificuldades na sua aplicação sob a ótica de enfermeiros. Revista Eletrônica Acervo Saúde, 2019; 11: 8.

3. CAMPOS NPS, et al. Dificuldades na implementação da sistematização de enfermagem. Revista Saúde em Foco, 2017.

4. CARLOS AMM, et al. liderança no ambiente hospitalar diferenças entre enfermeiros assistências e enfermeiros gerentes. Enferm. Foco, 2019; 10(6): 143-148.

5. CORDEIRO TLR, et al. Prontuário eletrônico como ferramenta para a sistematização da assistência de enfermagem no serviço de urgência/emergência: percepção dos enfermeiros. Rev Espaço para a Saúde, 2019; 20(2): 30-41.

6. FERREIRA VHS, et al. Contribuições e desafios do gerenciamento de enfermagem hospitalar: evidências científicas. Rev Gaúcha Enferm. 2019; 40: 20180291

7. GOMES ATL, et al. Safety of the patient in an emergency situation: perceptions of the nursing team. Rev. Bras. Enferm, 2019.

8. LATORRACA COC, et al. Busca em bases de dados eletrônicas da área da saúde: por onde começar. Diagn Tratamento,2019; 24(2): 59-63

9. MALVESTIO MAA, et al. Enfermagem em práticas avançadas no atendimento pré-hospitalar: oportunidade de ampliação do acesso no brasil. Enferm. Foco 2019; 10(6): 157-164.

10. MARIA MA, et al. Sistematização da assistência de enfermagem em serviços de urgência e emergência: viabilidade de implantação. Rev. bras. enferm., 2012; 65(2): 297-303.

11. MARCOS ACA, et al. Percepção da equipe de enfermagem quanto à sistematização da assistência de Enfermagem em um serviço de emergência psiquiátrica. Rev Min Enferm. 2016.

12. MARSICO EFC, SILVA SE. Os desafios encontrados na Sistematização da Assistência de Enfermagem em instituições hospitalares brasileiras. Enfermagem Brasil. 2012; 11(2).

13. MEDEIROS AL, et al. Desvelando dificuldades operacionais na sistematização da assistência de enfermagem através da Grounded Theory. Revista Eletrônica de Enfermagem. 2013; 15(1): 44-53.

14. MIRANDA CA, et al. Opinião de enfermeiros sobre instrumentos de atendimento sistematizado a paciente em emergência. Revista Rene. 2012; 13(2): 396-407.

15. MOHER D, et al. Principais itens para relatar Revisões sistemáticas e Meta-análises: A recomendação PRISMA. Epidemiol. Serv. Saúde, 2015; 24(2): 335-342

16. NICOLAU S, et al. Implementação da Sistematização da Assistência de Enfermagem (SAE) no Serviço de Atendimento Móvel de Urgência (SAMU). Rev. J. res.: fundam. care. online, 2019.

17. OLIVEIRA RJT, et al. Care management in nursing within emergency care units. Investigacion y educacion en enfermeria, 2015; 33(3): 406-414.

18. PAIXAO TCR, et al. Dimensionamento de enfermagem em sala de emergência de um hospital-escola. Rev. esc. enferm, 2015.

19. PINTO DM, et al. Utilização Da Sistematização Da Assistência De Enfermagem Em Serviço De Emergência: Vivência Dos Enfermeiros. REPENF - Rev. Parana. Enferm. 2021; 4(1): 96-103.

20. REIS GAX, et al. Dificuldades para implantar estratégias de segurança do paciente: perspectivas de enfermeiros gestores. Rev Gaúcha Enferm, 2019.

21. SANTOS WN. Sistematização da assistência de enfermagem: o contexto histórico, o processo e obstáculos da implantação. Rev. J Manag Prim Health Care, 2014.

22. SERRA HHN, et al. Implementation of risk-rated host in an emergency care unit. REVISA, 2019; 8(4): 484-95.

23. SOARES MI, et al. Sistematização da assistência de enfermagem: facilidades e desafios do enfermeiro na gerência da assistência. Esc. Anna Nery, 2015; 19(1): 47-53.

24. SOKOLSKI BL, et al. Desafios da enfermagem para atuação em urgência e emergência. Saúde Meio Ambiente, 2019; 8: 207-218

25. SOUSA LMM, et al. A metodologia de revisão integrativa da literatura em enfermagem. Revista investigação em enfermagem, 2017: 17-26.

26. SOUZA NR, BERALDO RAS. Desafios na implantação da sistematização da assistência de enfermagem nos setores de urgência. Rev enferm UFPE on line, 2015. 